\title{
Precision Single Mode Fiber Integral Field Spectroscopy with the RHEA Spectrograph
}

\author{
Adam D. Rains ${ }^{\mathrm{a}}$, Michael J. Ireland ${ }^{\mathrm{a}}$, Nemanja Jovanovic ${ }^{\mathrm{b}, \mathrm{c}}$, Tobias Feger ${ }^{\mathrm{c}}$, Joao Bento ${ }^{\mathrm{a}}$, \\ Christian Schwab ${ }^{\mathrm{c}, \mathrm{d}}$, David W. Coutts ${ }^{\mathrm{c}}$, Olivier Guyon ${ }^{\mathrm{b}, \mathrm{e}, \mathrm{f}, \mathrm{g}}$, Alexander Arriola ${ }^{\mathrm{c}, \mathrm{h}}$, and Simon \\ Gross ${ }^{\mathrm{c}, \mathrm{h}}$ \\ ${ }^{a}$ Research School of Astronomy and Astrophysics, Australian National University, Canberra, \\ ACT 2611, Australia

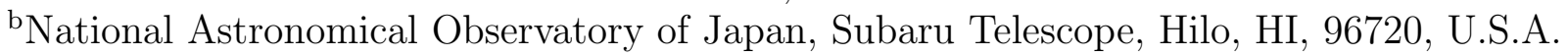 \\ ${ }^{c}$ Department of Physics and Astronomy, Macquarie University, NSW 2109, Australia \\ ${ }^{d}$ Australian Astronomical Observatory, 105 Delhi Rd, North Ryde NSW 2113, Australia \\ eSteward Observatory, University of Arizona, Tucson, AZ, 85721, U.S.A. \\ ${ }^{\mathrm{f}}$ College of Optical Sciences, University of Arizona, Tucson, AZ 85721, U.S.A. \\ ${ }^{\mathrm{g}}$ Astrobiology Center of NINS, 2-21-1, Osawa, Mitaka, Tokyo, 181-8588, Japan \\ ${ }^{\mathrm{h}}$ Centre for Ultrahigh bandwidth Devices for Optical Systems (CUDOS)
}

\begin{abstract}
The RHEA Spectrograph is a single-mode echelle spectrograph designed to be a replicable and cost effective method of undertaking precision radial velocity measurements. Two versions of RHEA currently exist, one located at the Australian National University in Canberra, Australia (450 - 600nm wavelength range), and another located at the Subaru Telescope in Hawaii, USA (600 - $800 \mathrm{~nm}$ wavelength range). Both instruments have a novel fibre feed consisting of an integral field unit injecting light into a $2 \mathrm{D}$ grid of single mode fibres. This grid of fibres is then reformatted into a 1D array at the input of the spectrograph (consisting of the science fibres and a reference fibre capable of receiving a white-light or xenon reference source for simultaneous calibration). The use of single mode fibres frees RHEA from the issue of modal noise and significantly reduces the size of the optics used. In addition to increasing the overall light throughput of the system, the integral field unit allows for cutting edge science goals to be achieved when operating behind the $8.2 \mathrm{~m}$ Subaru Telescope and the SCExAO adaptive optics system. These include, but are not limited to: resolved stellar photospheres; resolved protoplanetary disk structures; resolved Mira shocks, dust and winds; and sub-arcsecond companions. We present details and results of early tests of RHEA@Subaru and progress towards the stated science goals.
\end{abstract}

Keywords: Spectrograph, Radial Velocity, Optical fibers, Fiber injection, diffraction-limited spectrograph, high resolution, integral-field unit

\section{INTRODUCTION}

RHEA, the Replicable High-resolution Exoplanet and Asteroseismology Spectrograph, is a compact single-mode echelle spectrograph designed to be a replicable and cost-effective method of undertaking precision radial velocity measurements. The instrument has a novel fiber feed consisting of a diffraction-limited IFU injecting light into a 2 dimensional grid of Single Mode Fibers (SMF). The main objective of the RHEA program is to conduct long term RV measurements from a number of sites on automated small telescopes, taking advantage of the instrument's low cost, technically mature design and the lack of competition for time on smaller telescopes. For such applications RHEA's fiber feed serves to improve throughput, particularly in the presence of atmospheric turbulence. However, when placed behind an extreme adaptive optics system on an 8-m class telescope, the instrument becomes capable of spatially resolving targets at the 8 milli-arcsecond level at a high spectral resolution, opening up a wide range

Further author information: (Send correspondence to M.Ireland)

M.Ireland: E-mail: michael.ireland@anu.edu.au, Telephone: +61 (0)2 61250288

Ground-based and Airborne Instrumentation for Astronomy VI, edited by Christopher J. Evans, Luc Simard, Hideki Takami Proc. of SPIE Vol. 9908, 990876 · (c) 2016 SPIE · CCC code: 0277-786X/16/\$18 · doi: 10.1117/12.2233743 
of science cases unique to the instrument. This will enable the direct detection of the accretion from forming exoplanets, will detect scattered light from protoplanetary disks resolved simultaneously spatially and in velocity, and will create velocity-resolved movies of convection cells and mass loss processes from giant stars nearing the ends of their lives.

The Subaru Telescope in Hawaii, alongside the Subaru Coronographic Extreme Adaptive Optics (SCExAO) System, ${ }^{1}$ make this possible. RHEA@Subaru has now been fully commissioned, following SCExAO engineering time in February and March 2016 and is the first optical (R-band) IFU behind an Extreme Adaptive Optics system, with spectral and spatial resolutions of $\sim 60,000$ and 8 milli-arcseconds respectively. The only other comparable instrument is SINFONI ${ }^{2}$ on the VLT, which operates in the $\mathrm{J}, \mathrm{H}, \mathrm{K}$ and $\mathrm{H}+\mathrm{K}$ bands (with spectral resolutions of 20,000,30,000, 40,000 and 15,000 respectively). Critically though, the smallest spatial resolution possible with SINFONI is $12.5 \times 25$ milli-arcseconds - insufficient to cover the range of targets RHEA's resolution enables. RHEA's small IFU does not make the instrument a discovery machine. It does however provide excellent follow-up of isolated objects, or observations of objects barely resolved with an $8 \mathrm{~m}$ telescope. Table 1 gives an overview of the key instrument parameters.

\begin{tabular}{c|l} 
Table 1. Design specifications of the RHEA@Subaru spectrograph and IFU. \\
\hline Spatial Resolution & 8 milli-arcsec \\
Spectral Resolution & R $\sim 60,000$ \\
Total Field of View & $\sim 4$ arcsec \\
Instantaneous Field of View & 40 milli-arcsec \\
IFU Elements & 9 (with dithering capability) \\
Spectrograph Total Efficiency & $35 \%$ \\
Injection Unit Efficiency & Strehl $\times 0.6$ \\
Wavelength Range & $600-800 \mathrm{~nm}$ \\
\hline
\end{tabular}

Development and support of RHEA@Subaru and associated software (e.g. control software, data reduction and analysis pipeline software) is ongoing. Should the RHEA module become available for general use, the RHEA@Subaru team are more than willing to work with the Subaru community to help acquire and reduce data. See Ref. 3 for details on current SCExAO developments (including RHEA). Refs. 4, 5, \& 6 should be consulted for a more detailed overview of the RHEA spectrograph and how it applies to exoplanets and asteroseismology.

In this paper we first detail the science possible with RHEA@Subaru in Section 1.1. In Section 2 we describe the design of the instrument and the novel fibre feed employed. Section 3 discusses preliminary results and performance. Section 4 gives and overview of the future of RHEA@Subaru and planned improvements. Finally, we summarize this paper in Section 5.

\subsection{Feasible Science with RHEA@Subaru}

As previously mentioned, RHEA@Subaru opens up unique science opportunities. The following sections go into these in more detail.

\subsubsection{Accreting Protoplanets}

In recent years, several young wide separation giant planets have been found to have disks and are accreting. Some of these are wide separation giant planets (e.g. GSC6214b $\mathrm{b}^{7,8}$ ), while others are still embedded in their parent protoplanetary disks. ${ }^{9}$ The H- $\alpha$ emission from the accretion shock is extremely bright - for example, GSC6214b has $10^{-15} \mathrm{erg} \mathrm{cm}^{-2} \mathrm{~s}^{-1} \AA^{-1}$, which was detected by $\mathrm{HST}^{7}$ with a relatively broad filter in H- $\alpha$ with a very high signal to noise of 300 in 40 minutes of exposure time. However, the velocity width, critical to estimating the mass of this young exoplanet, has not been directly measured. Assuming a $100 \mathrm{~km} \mathrm{~s}^{-1}$ width, the contrast in the core of the $\mathrm{H}-\alpha$ line would only be approximately 1 magnitude. Other exoplanets with extremely red colors, e.g. HD $95086 \mathrm{~b}^{10}$ may also have a circumplanetary disk, again which could show $\mathrm{H}-\alpha$ emission. SCExAO with a deployable IFU would be the ideal capability to follow up these kinds of objects, so long as the overall efficiency is close to that designed and Strehl remains high. 


\subsubsection{Resolved Protoplanetary Disk Structures}

Scattered light in young disks is an important probe of the disk surface layers, which are particularly sensitive to variations in the disk scale height [e.g. Ref. 11]. In a flaring disk model, the disk surface brightness is proportional to $r^{-12 / 7}$, meaning that inner regions of the disk are much brighter than the typically resolved outer regions of the disk. For example, the disk surface scatters a fraction of $10^{-3}$ of the light from the star between 1 and $2 \mathrm{AU}$. Any gap or disk substructure produces an inner wall that is much brighter than this canonical disk model [e.g. Ref. 12] by up to a factor of 10 .

Polarized intensity has been the so-far standard way to reduce the influence of the speckles of the telescope point-spread function. For typical grain size distributions, polarized intensity is approximately a factor of 5-10 lower than total intensity, which can make these observations challenging. An alternative is to velocity-resolve the emission. With a stellar rotation velocity of $\sim 10 \mathrm{~km} \mathrm{~s}^{-1}$, the minimum spectral resolution needed to separate disk from stellar emission is $\mathrm{R} \sim 30,000$.

Several young stars are ideal candidates for this kind of work. The prototype T Tauri star, T Tauri, is well within the magnitude range of SCExAO, and with a moderate $\sim 20 \mathrm{~km} \mathrm{~s}^{-1} v \sin (i)$ and mass $\left(3 \mathrm{M}_{\odot}\right)$ will have a scattered light disk resolved by 1-2 IFU elements. Many of the other, most promising resolved disk candidates are not easily observable by SCExAO, due to their brightness. The most promising candidates include DM Tau, which has $m_{V}=14$ and $m_{I}=11.5$ - at the limit of good SCExAO correction. The brightest T Tauri stars in Taurus (the closest star forming region accessible in the Northern Hemisphere) include AB Aur, SU Aur, HD 283572 and RY Tau, all of which have high $\mathrm{V} \sin (i)$ values. This means that $\mathrm{R} \sim 10,000$ is all that is required, and also that the scattered light at large velocity offsets is resolved.

\subsubsection{Resolved Stellar Photospheres and Molspheres}

The largest stellar photospheres are directly observable from Subaru using SCExAO. The very largest stars accessible from Subaru include Betelgeuse (Alpha Orionis) at 50 milli-arcseconds, Mira (Omicron Ceti) at $\sim 50$ milli-arcseconds, Antares (Alpha Scorpii) at $\sim 40$ milli-arcseconds, Arcturus (Alpha Bootis) at $\sim 21$ milliarcseconds and Aldebaran (Alpha Tauri) at $\sim 19$ milli-arcseconds. These giant stars all lose mass through a complex process where there is an interplay between a hot $(\sim 10,000 \mathrm{~K})$ corona and a cool $(\sim 2000 \mathrm{~K})$ slow $\left(\sim 10 \mathrm{~km} \mathrm{~s}^{-1}\right)$ molecular wind [e.g. Ref. 13]. These states can not co-exist, so asymmetries of some kind are expected. Resolving the photosphere in lines and molecular bands will enable the multi-dimensional structure of these near-photospheric regions to be imaged. Upwelling and downwelling velocities on the surface are also of order a few $\mathrm{km} \mathrm{s}^{-1}$, which can be separated at sufficient resolution. A single image of a stellar photosphere would be the highest resolution direct measurement of convection in a star other than the sun. See Refs. 14, 15, 16 \& 17 for examples of past research into resolved stellar photospheres and outflows. Time-dependent observations showing a consistent "upwelling" on one side of the star and a "downwelling" on the other side would be one of the only possible clear indication of the rotational periods of these giant stars - a critical parameter for magnetic field generation and mass loss in the final stages of evolution which is inaccessible by other means.

\subsubsection{Resolved Mira Shocks, Dust and Winds}

Mass-losing red giants, in particular Mira variables, are the subject of extensive observational study today [e.g. Ref. 18]. At the heart of mass loss is kinematics: the determination of which parcel of gas is moving outwards, and which is moving inwards. This is easily resolvable by RHEA@Subaru - for example, TiO bands with line spacing more than $10 \mathrm{kms}^{-1}$ (as occurs for spectral regions with hundreds of lines) will have a image in the blue-shifted part of the absorption that looks like a ring, with a dark hole in the center. A high-SNR image does not have to be formed at every spectral channel, as many lines probe one physical phenomena.

\subsubsection{Sub-arcsecond Companions}

Objects such as Mira B are well resolved, but are significantly fainter than the Mira A photosphere so are extremely difficult to study. Mira AB is the nearest wind accretion binary and the easiest to study, but it is still controversial as to whether Mira B is a main sequence star or white dwarf. If Mira B is a white dwarf, then it is either extremely under luminous or accretes well below the rate expected from Bondi-Hoyle accretion or dynamical models. If Mira B is a main sequence star, then the maximum wind velocity is extremely high. Even 
at moderate Strehl ratios, Mira B could be separated form Mira A so long as the spectra are different. Just a single spectrum of Mira B at 600-800 nm could determine if it is a white-dwarf or a main-sequence star.

\subsection{Science Case Instrument Specifications}

\subsubsection{Simultaneous Operation}

As many of the key science targets for RHEA are also science targets for other programs using e.g. Vampires ${ }^{19}$ or the SCExAO science camera, it is essential that RHEA is able to run completely in parallel without affecting the other (possibly primary) science program. This must include a complete suite of internal calibration sources.

\subsubsection{Wavelength Range}

The key wavelength rage is around $\mathrm{H}-\alpha(600-700 \mathrm{~nm})$, where there are also key molecular TiO absorption bands for the giant star science cases. Increased wavelength coverage to $800 \mathrm{~nm}$ is desirable to access higher Strehl ratios, and also a line of potassium which may probe higher regions of stellar atmospheres.

\subsubsection{Spectral Resolution}

The minimum spectral resolution varies by science case. For the accreting protoplanets and protoplanetary disk science cases, the minimum resolving power is 15,000 , which is enough to barely resolve lines in a rapidly rotating young star $\left(20 \mathrm{~km} \mathrm{~s}^{-1}\right)$ or clearly resolve a $\mathrm{H}-\alpha$ emission peak. For giant stars, individual molecular lines in typical bands begin to be resolved at $\mathrm{R} \sim 10,000$, but the ideal spectral resolution would be enough to resolve the turbulent velocity in the stellar atmosphere, i.e. $\mathrm{R} \sim 150,000 .{ }^{20}$ A resolution around $\mathrm{R} \sim 60,000$ is an excellent compromise resolution for these science cases.

\subsubsection{Spatial Resolution and Field of View}

Given that RHEA will generally be observing barely resolved objects, only a small number of spaxels are needed in order to resolve first-order effects such as stellar rotation, or centroid of a line as a function of velocity. For the largest stellar photospheres, for example Betelgeuse ( $\alpha$ Ori), a larger field of view is needed.

The instantaneous field of view of $40 \times 40$ milli-arcsec is well matched to a typical stellar photosphere. Critically, the IFU must have the capability for dithering around the field of view in open or closed loop (i.e. knowing both where the IFU is in the image plane while imaging the field). A typical dither pattern is shown in Figure 1.

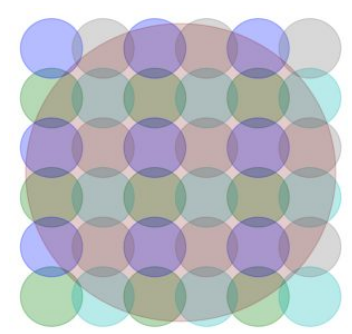

Figure 1. A schematic of a 4-point dither pattern for the RHEA IFU, with the 9 IFU elements for each dither in blue, gray, cyan and green colors. A 48 milli-arcsec diameter stellar photosphere is overlayed.

\subsubsection{Sensitivity}

As a typical exposure with RHEA@Subaru will have to be reasonably short, because of the need to dither the mini-IFU probe for many science goals. To calculate anticipated sensitivity, we will be conservative, and assume a RHEA efficiency of $30 \%$, a fiber effective diameter of 10 mas, a complete Telescope and AO system efficiency of $20 \%$, a Strehl ratio of $10 \%$ and a fiber coupling efficiency of $60 \%$. For a point source, this would give an overall efficiency of $0.4 \%$, or an efficiency per fiber of $1.4 \times 10^{-4}$ for Betelgeuse. In turn, this gives $\sim 10^{5}$ photons per fiber per spectral pixel (0.4 resolution elements) per $5 \mathrm{~s}$ exposure for Betelgeuse (near saturation), or 1000 photons per fiber per spectral pixel for omi Cet near minimum light per $15 \mathrm{~s}$ exposure. For a resolved stellar photosphere, omi Cet near minimum has roughly the lowest surface brightness of any stellar object in the sky - it 
is clear that sensitivity will be adequate for any stellar photosphere science. For e.g. Mira B (assumed $m_{R}=10$ ), exposure times of 1 minute would be sufficient to place the spectrum above the readout noise.

For the other science cases, sensitivity is more critical, and will vary rapidly with Strehl ratio. Achieving a $5-\sigma$ point-source detection per resolution element at $m_{R} \sim 14$ in $1000 \mathrm{~s}$ of integration time is more than enough for all science cases mentioned above.

\section{INSTRUMENT DESCRIPTION}

\subsection{The RHEA Spectrograph}

The spectrograph for RHEA@Subaru is nearly identical to the version of RHEA described in Refs. $4,5 \&$ 6. Compact re-imaging optics converts the plate-scale to 9 milli-arcsec $\mathrm{mm}^{-1}$, at which point light is injected into a $3 \times 3$ array of fibers with $1 \mathrm{~mm}$ spacing. Diffraction-limited achromatic SMF injection is achieved with active alignment during initial assembly of the all-glass bonded injection unit made of silica and N-SF11. This mini-IFU can be positioned anywhere within a 7 arcsec field of view, with the central \pm 1 arcsec field being unvignetted and a multi-mode fiber with calibration sources placed in one corner of the 7 arcsec field. The 9 IFU elements are arranged in a linear slit at the spectrograph input, together with a simultaneous reference fiber. The spectrograph itself is a prism cross-dispersed near-littrow echelle spectrograph with a $1 / e^{2}$ beam width of $\sim 13 \mathrm{~mm}$ and a resolving power of 75,000 to 50,000 over the wavelength range 550 to $800 \mathrm{~nm}$. The spectrograph optics are thermally controlled to a $<5 \mathrm{mK}$ precision in a lab environment or a $\sim 10 \mathrm{mK}$ precision in a typical telescope environment. Peak slit-to-detector efficiency is $\sim 40 \%$ : high for an echelle spectrograph due to the design's simplicity. For RHEA@Subaru, the only differences to existing publications are that:

- The spectrograph input is a multi-fiber input (described below).

- The vacuum chamber is not intended for operation at Subaru, but is still installed as part of the spectrograph.

- There are two cross-dispersion prisms instead of one, to accommodate a longer slit width and longer wavelengths.

- A different angle on the grating, to tune the wavelength to $600-800 \mathrm{~nm}$.

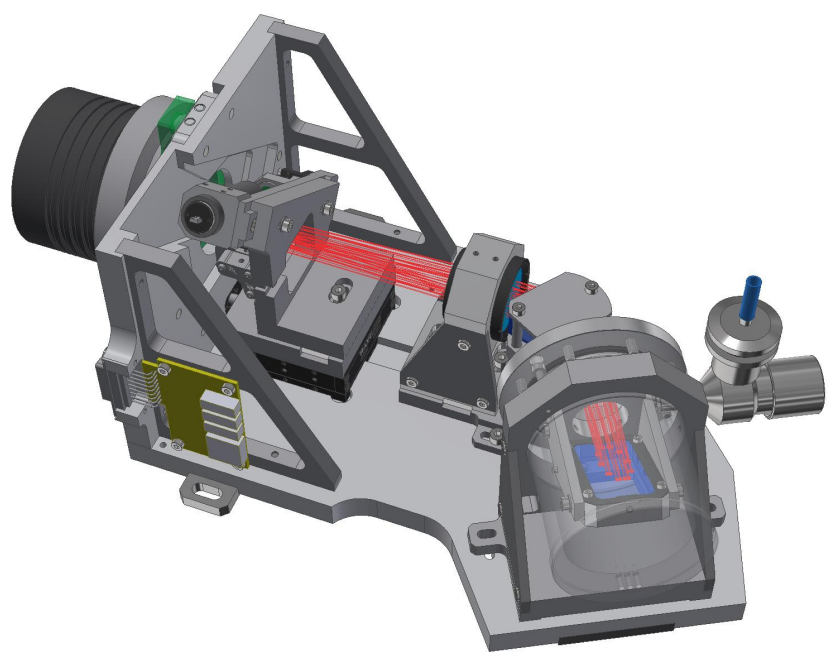

Figure 2. A solid model of the spectrograph opto-mechanics as optimised for Subaru. The key difference to the spectrograph presented in Ref. 4 is the use of two N-SF5 prisms to obtain sufficient cross-dispersion at the RHEA@Subaru wavelength range. 
A solid model of the RHEA@Subaru spectrograph is shown in Figure 2. Information on the resolution, efficiency and stability tests of the visible-light version of RHEA can be found in Ref. 5. The longer-wavelength RHEA@Subaru has similar performance, with tests ongoing.

\subsection{Fibre Feed}

RHEA@Subaru is designed to take an array of 9 fibers in a square array (IFU), and place them in a linear array at the spectrograph input (V-groove). A 10th fiber (separated by a 1-fiber gap) can also be illuminated by either a white-light source or a neon reference source. These same sources can be injected into the RHEA input by a large multi-mode fiber in a fixed position, simply by commanding the IFU to move to the multi-mode fiber location. This enables calibration of all fibers in the spectrograph slit. We will first describe the feed end, then the slit end.

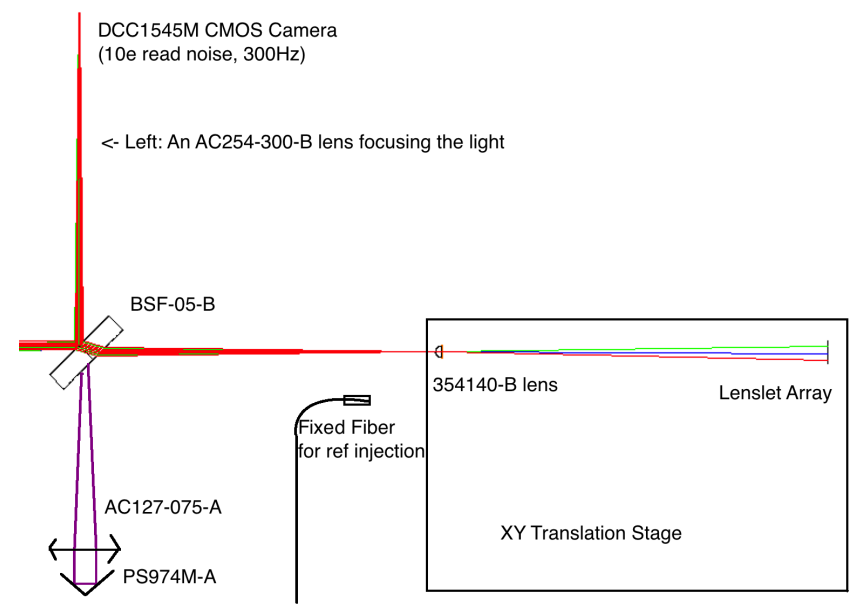

Figure 3. A sketch of the injection optics for RHEA@Subaru. Not shown is the $300 \mathrm{~mm}$ focusing lens to the left, which is on a z-axis (focusing) stage.

The layout of the injection optics as installed at SCExAO is shown in Figures 3. The key parameters of the feed are as follows:

- $D_{1}=7920 \mathrm{~mm}$ Subaru pupil diameter.

- $D_{2}=7.2 \mathrm{~mm}$ input pupil in SCExAO.

- $f_{1}=300 \mathrm{~mm}$ focusing lens 1 (AC254-300-B)

- $f_{2}=1.45 \mathrm{~mm}$ re-focusing lens $2(354140-\mathrm{B})$

- $\Delta x=1 \mathrm{~mm}$ lenslets in final image plane (OKOTech APO-Q-P1000-F4,64 (633)).

- A target $\Delta \theta=7.7 \times 10^{-8}$ radians (16 milli-arcsec) on-sky angular separation between lenslets. This gives Nyquist sampling after a $2 \times 2$ drizzle pattern.

The target value of 16 milli-arsec per lenslet means that the following distance from lens 2 to the microlens array applies:

$$
d=\frac{D_{2} f_{2} \Delta x}{D_{1} f_{1} \Delta \theta}
$$

This gives $57 \mathrm{~mm}$ between lens 2 and the lenslet array. The lenslet array is designed to take the common pupil (located $\sim 1 \mathrm{~mm}$ to the right of the $1.45 \mathrm{~mm} 354140-\mathrm{B}$ lens) and focus this on to 9 fibers separately. The feed optics are shown in Figure 3 and the IFU (during fabrication) is shown in Figure 4. 
An active optical alignment procedure was used during the IFU assembly in order to align each fiber with its respective microlens. Fibers were back-illuminated with a laser source and positioned using a six-axis stage, using a camera to image the common pupil plane of each fiber. With this single-camera setup, a fiducial fiber was illuminated and used as a reference to ensure the output of all fibers overlapped in the pupil plane.
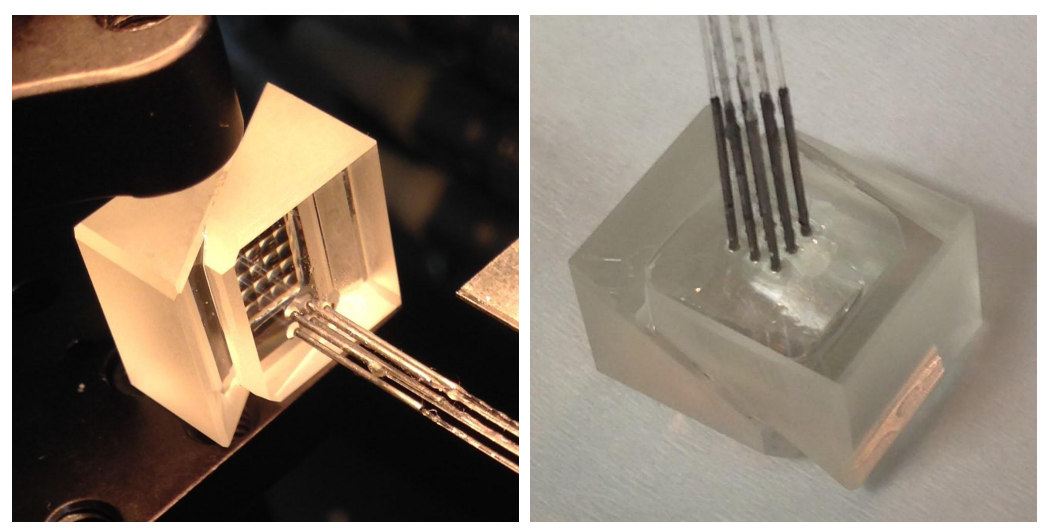

Figure 4. The IFU during the fiber gluing procedure. The elements are: an APO-Q-P1000-F4,64 (633) OKOTech microlens array (fused silica, $2.12 \mathrm{~mm}$ radius of curvature, $4.64 \mathrm{~mm}$ focal length), a PS870 unmounted anamorphic prism pair from Thorlabs, and 630-HP SMFs in metal ferrules of outer diameter $0.4 \mathrm{~mm}$ ). Prisms were used to precisely (and easily) locate the fiber tips at the focus of the microlens array (as opposed to the more complex procedure using a single piece of glass). Each element is held together with index matching NOA 61 UV curing glue. The IFU was assembled at the Australian National Fabrication Facility - OptoFab precision alignment and assembly facilities in Sydney after a series of prototyping efforts.

The slit end of the fiber feed needs to take the $9+1$ fibers and turn them into a slit with $\mathrm{f} / 18\left(1 / \mathrm{e}^{2}\right)$ focal ratio and a common pupil near infinity (at $\sim 150 \mathrm{~mm}$ distance). This is accomplished using the setup shown in Figure 5. Figure 6 shows a $5 \mathrm{~ms}$ exposure of the fiber tips in the V-groove (including the reference fiber) through the completed fiber cable (IFU through to slit optics) with the IFU illuminated by a $740 \mathrm{~nm}$ source.
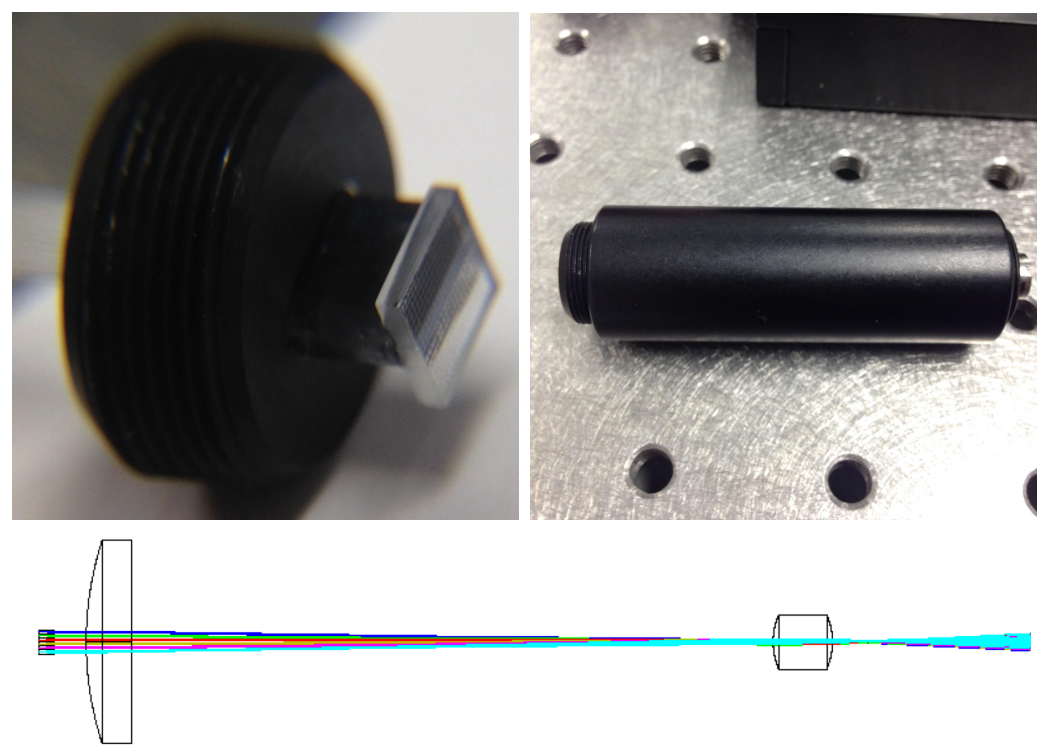

Figure 5. An overview of the slit formatting optics for RHEA@Subaru. The elements are: a V-groove array from OzOptics with $250 \mu \mathrm{m}$ spacing (assembled in the lab at ANU), a SUSS 18-00030 microlens array (fused silica, $0.297 \mathrm{~mm}$ radius of curvature), a LA1304-B $40 \mathrm{~mm}$ focal length lens to form a pupil, and a N414TM-B, $3.3 \mathrm{~mm}$ focal length slit-forming lens - all contained in a 0.5 inch diameter lens tube. The lowermost panel shows a Zemax model of the optics. 
Figure 6. Illuminated fibers (740 nm source, $5 \mathrm{~ms}$ exposure) through slit forming optics. The simultaneous reference fiber is on the left, with the nine science fibers following after a single fiber gap.

\subsection{Data Reduction Pipeline}

Data reduction will be undertaken with a custom pipeline written specifically with multiple fiber echelle spectrographs in mind. It uses methods described by Ref. 21 to account for the tightly packed nature of the fibers and their overlapping profiles, minimizing crosstalk and reducing noise. Data (in this case nine science fibers plus the reference source fiber) are calibrated for basic instrumental effects (e.g. dark/flat correction) before fluxes are extracted using a wavelength model of dispersion on the CCD. A reference spectrum, typically a combination of a number (e.g. 10) subsequent observations from the same star (though spectra from a star of a similar spectral type can also be used) is then used to calculate the RVs for each of the science spectra. The resultant RVs are then corrected for the barycentric velocity and calibrated for instrumental drift using the neon reference RVs. The nature of the pipeline means that individual spectral features are not required to be identified in order to extract RVs as the RVs are computed instead through direct comparison between entire spectral orders. The performance of the pipeline has been verified with test stellar data taken during SCExAO engineering nights in February and March of 2016.

\section{PRELIMINARY RESULTS}

On-sky observations were undertaken during the February and March 2016 SCExAO runs, with the focus on both testing the instrument and observing resolved stars. Conditions during the runs were not sufficient to resolve the latter targets to an acceptable degree, and low-order modes were poorly corrected, resulting in low throughput. Nonetheless, the nights served as an excellent test of the instrument, observational procedures, control software and data reduction pipeline.

The fiber cable was found to be afflicted by modal noise and cross-talk due to the closely packed fibers in the V-groove having very similar path lengths, as shown in Figure 7 and Figure 8. Due to optical aberrations (especially chromatic defocus), the electric fields of neighboring fibers overlap somewhat on the detector. This leads to constructive and destructive interference, depending on the fiber path length difference. For a small path length difference $\left(<\lambda^{2} / \delta \lambda\right)$ either constructive or destructive interference occurs on each pixel, and is sensitive to injection conditions, fiber cable temperature and fiber cable temperature gradients.

Figure 9 shows a spectrum of Arcturus, where the modal noise is less apparent when considering all fibers together, thus showing the spectral resolution of the instrument. 


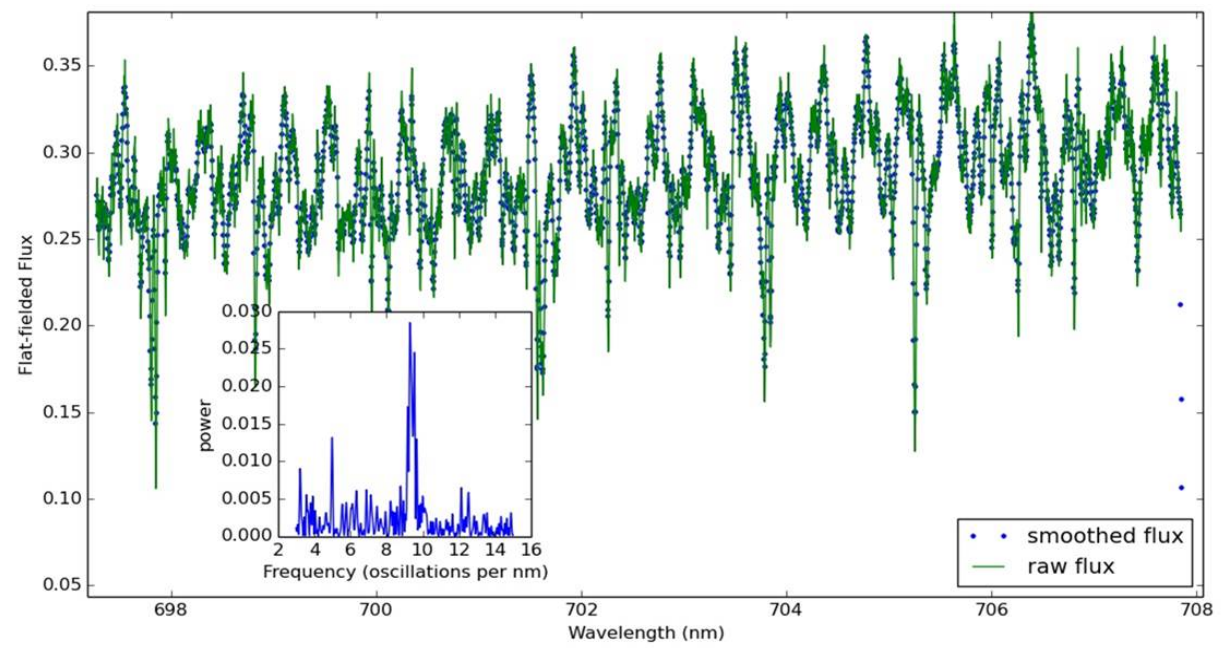

Figure 7. Flat fielded flux (arb. units) as a function of wavelength (nm). Modal noise is visible in each order for this exposure of Arcturus.
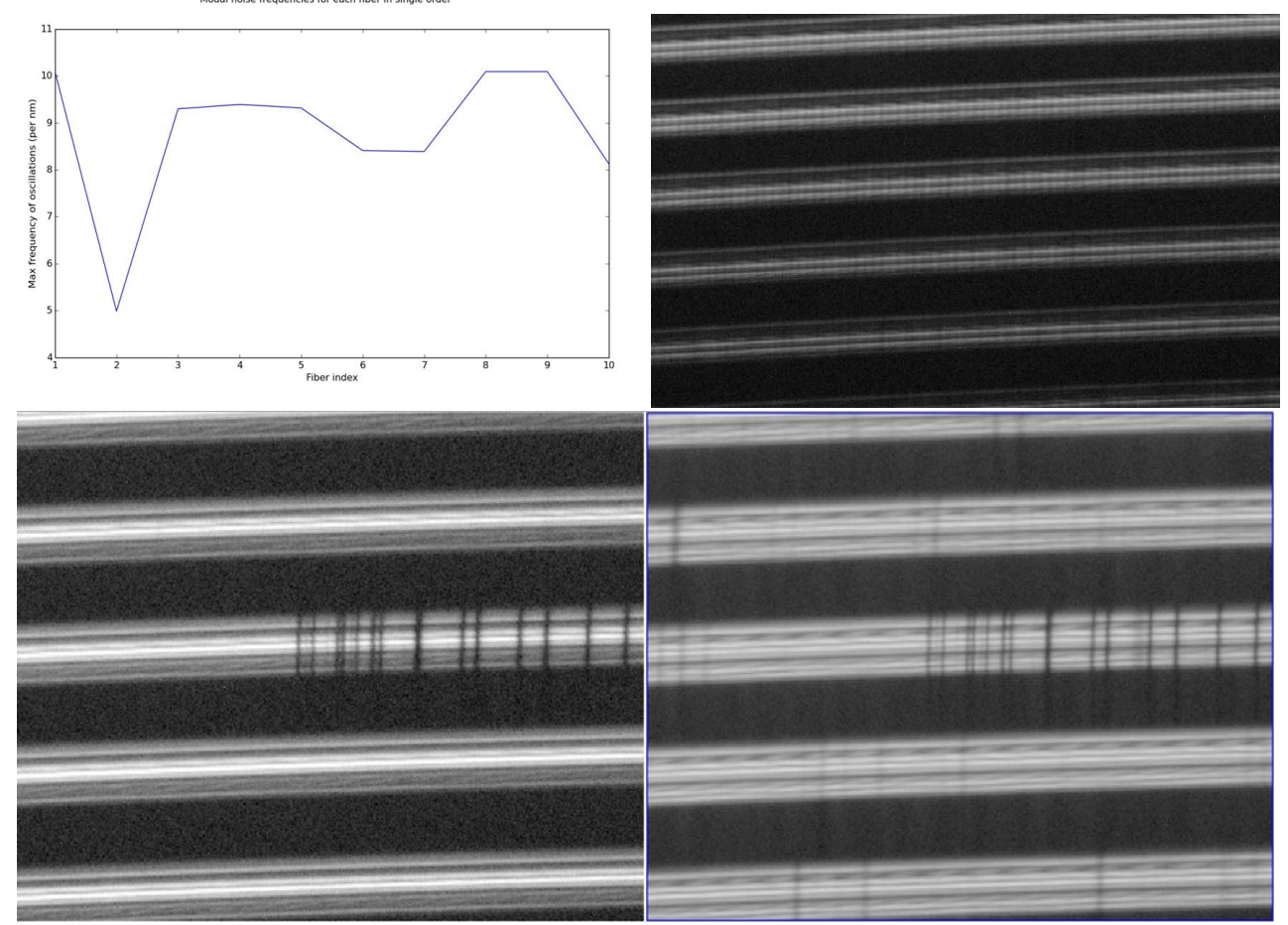

Figure 8. Modal noise dominated by interaction between neighboring fibers Frequency therefore varies for each fiber in a single order. Clockwise from top left: Modal noise frequencies for each fiber [A]; supercontinuum source flat field [B], Arcturus with fiber agitator OFF [D]; Sirius with fiber agitator ON [C]

\section{FUTURE WORK}

The development and commissioning process for RHEA@Subaru revealed a number of issues to be resolved before the instrument can meet its full potential. These are detailed in the following section. 


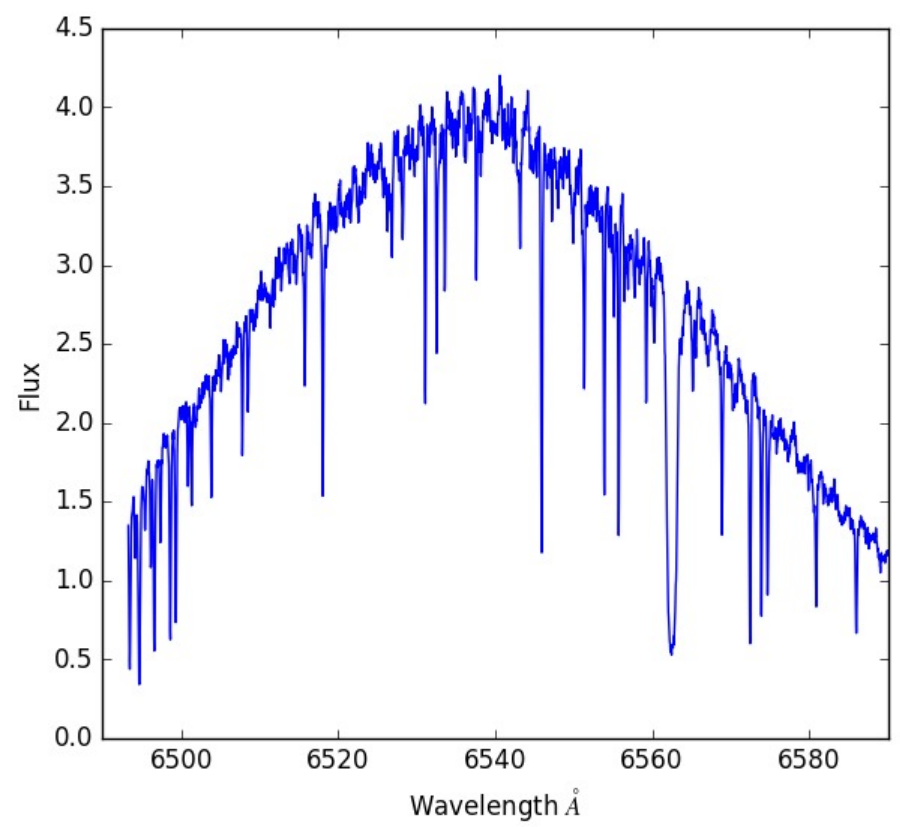

Figure 9. Flux (arb. units) as a function of wavelength $(\AA)$ for a raw example spectrum of Arcturus, with a 30 s exposure. The modal noise in this case is barely visible with all fibers are added together.

\subsection{Modal Noise}

To address the issue of modal noise, a replacement cable for RHEA@Subaru will be fabricated, with the intent to improve flaws identified in the original procedure and reduce the influence of such noise on data taken. When fabricating the IFU a more robust two-camera optical alignment procedure will be employed. This will allow the simultaneous imaging of the common pupil plane and the surface of the microlens array - a procedure already successfully employed for the cable of another RHEA iteration. Additionally, changes will be made to the fiber gluing procedure, making use of custom fabricated glass ferrules (with tighter inner diameters) transparent to UV light, and a glass mask to locate each fiber and provide greater structural integrity when gluing each fiber.

If the path length difference between neighboring fibers is increased, then the pattern in wavelength space of constructive and destructive interference is unresolved. Thus to combat modal noise, path length differences will be inserted in fibers neighboring each other in the V-groove in the form of small loops in the fiber. Modal noise is most significant between neighbors, so it will not be necessary for each fiber to have a unique path length having fibers alternate between having a single loop and having no loop will be a sufficient measure.

\subsection{Software}

Both the control software and data reduction pipeline are in a continuous state of development and will see improvements in both reliability and efficiency by the next Subaru observing run.

\subsection{Observing Conditions}

The data taken during the February and March 2016 SCExAO observing runs were sufficient to serve as a test for the hardware, software and operating procedure, but the conditions were not sufficient to meet any of the science goals. For RHEA@Subaru to achieve the science it was designed for, seeing at the 8 milli-arcsecond level or better will be required. 


\section{SUMMARY}

The RHEA@Subaru module for SCExAO will be completely unique in the world, providing a spectral resolution more than 10 times higher than any adaptive optics fed IFU. This will enable a variety of science programs, including directly resolving convection cells and the mysterious wind-launching zones of giant stars. RHEA@Subaru has been successfully commissioned and utilized during the February and March 2016 observing runs. The instrument operated as designed, but several unforeseen issues, including the modal noise introduced by neighboring fibers and poor weather conditions prevented the instrument on making progress towards its science goals. Despite this, the data taken served as an excellent test for the custom software reduction pipeline, designed specifically for multiple-fiber echelle spectroscopy.

Following the run, plans are in place to replace the existing fibre cable and IFU feeding the instrument with a new version, incorporating a more robust fabrication procedure and steps to free the instrument of modal noise. With this change, alongside steady improvements to control software and the reduction pipeline, RHEA@Subaru will be able to become a productive, and more importantly unique in the world, instrument operating at the Subaru Telescope in Hawaii.

\section{ACKNOWLEDGMENTS}

This work was partially funded by the Australian Research Council Discovery Project DP120103751. We would like to thank Macquarie Engineering and Technical Services (METS) and the RSAA Manufacturing Workshop for their work in machining parts to complete the spectrograph assembly. Furthermore we thank Dr Benjamin Johnston, Dr Martin Ams and Alex Stokes from OptoFab for their assistance in constructing the fibre cable used for RHEA@Subaru, as well as OptoFab for use of the Nanosecond Laser Micro-Fabrication Facility, and Optical Device Characterisation Suite. Adam Rains acknowledges support through the RSAA Masters (Advanced) Scholarship from the Research School of Astronomy and Astrophysics and Australian National University. Most importantly we would like to thank the Subaru Telescope and the National Astronomical Observatory of Japan for granting us the opportunity to undertake this project and for their continued assistance throughout.

\section{REFERENCES}

[1] Jovanovic, N., Martinache, F., Guyon, O., Clergeon, C., Singh, G., Kudo, T., Garrel, V., Newman, K., Doughty, D., Lozi, J., Males, J., Minowa, Y., Hayano, Y., Takato, N., Morino, J., Kuhn, J., Serabyn, E., Norris, B., Tuthill, P., Schworer, G., Stewart, P., Close, L., Huby, E., Perrin, G., Lacour, S., Gauchet, L., Vievard, S., Murakami, N., Oshiyama, F., Baba, N., Matsuo, T., Nishikawa, J., Tamura, M., Lai, O., Marchis, F., Duchene, G., Kotani, T., and Woillez, J., "The Subaru Coronagraphic Extreme Adaptive Optics System: Enabling High-Contrast Imaging on Solar-System Scales," Publications of the Astronomical Society of the Pacific 127, 890-910 (Sept. 2015).

[2] Angerhausen, D., Krabbe, A., and Iserlohe, C., "Near-infrared integral-field spectroscopy of HD209458b," arXiv:astro-ph/0605315 , 62694S-62694S-9 (June 2006). arXiv: astro-ph/0605315.

[3] Jovanovic, N. and et al., "The SCExAO high contrast imager: transitioning from commissioning to science," in [SPIE 9909], Vol. 9909, 990934, SPIE Proceedings, Edinburgh, Scotland (2016).

[4] Feger, T., Bacigalupo, C., Bedding, T. R., Bento, J., Coutts, D. W., Ireland, M. J., Parker, Q. A., Rizzuto, A., and Spaleniak, I., "RHEA: the ultra-compact replicable high-resolution exoplanet and Asteroseismology spectrograph," in [SPIE 9147], Ramsay, S. K., McLean, I. S., and Takami, H., eds., Vol. 9147, 91477I, SPIE Proceedings, Montral, Quebec, Canada (July 2014).

[5] Feger, T. and et al., "Attaining ms-1 level intrinsic Doppler precision with RHEA, a low-cost single-mode spectrograph," Submitted (2015).

[6] Bento, J. and et al., "Performance and future developments of the RHEA single-mode spectrograph," in [SPIE 9908], Vol. 9908, 9908248, SPIE Proceedings, Edinburgh, Scotland (2016).

[7] Zhou, Y., Herczeg, G. J., Kraus, A. L., Metchev, S., and Cruz, K. L., "Accretion onto Planetary Mass Companions of Low-mass Young Stars," The Astrophysical Journal Letters 783, L17 (Mar. 2014).

[8] Ireland, M. J., Kraus, A., Martinache, F., Law, N., and Hillenbrand, L. A., "Two Wide Planetary-mass Companions to Solar-type Stars in Upper Scorpius," The Astrophysical Journal 726, 113 (Jan. 2011). 
[9] Sallum, S., Follette, K. B., Eisner, J. A., Close, L. M., Hinz, P., Kratter, K., Males, J., Skemer, A., Macintosh, B., Tuthill, P., Bailey, V., Defrre, D., Morzinski, K., Rodigas, T., Spalding, E., Vaz, A., and Weinberger, A. J., "Accreting protoplanets in the LkCa 15 transition disk," Nature 527, 342-344 (Nov. 2015).

[10] Galicher, R., Rameau, J., Bonnefoy, M., Baudino, J.-L., Currie, T., Boccaletti, A., Chauvin, G., Lagrange, A.-M., and Marois, C., "Near-infrared detection and characterization of the exoplanet HD $95086 \mathrm{~b}$ with the Gemini Planet Imager," Astronomy and Astrophysics 565, L4 (May 2014).

[11] Pohl, A., Pinilla, P., Benisty, M., Ataiee, S., Juhsz, A., Dullemond, C. P., Van Boekel, R., and Henning, T., "Scattered light images of spiral arms in marginally gravitationally unstable discs with an embedded planet," Monthly Notices of the Royal Astronomical Society 453, 1768-1778 (Oct. 2015).

[12] Thalmann, C., Mulders, G. D., Janson, M., Olofsson, J., Benisty, M., Avenhaus, H., Quanz, S. P., Schmid, H. M., Henning, T., Buenzli, E., Mnard, F., Carson, J. C., Garufi, A., Messina, S., Dominik, C., Leisenring, J., Chauvin, G., and Meyer, M. R., "Optical Imaging Polarimetry of the LkCa 15 Protoplanetary Disk with SPHERE ZIMPOL," The Astrophysical Journal Letters 808, L41 (Aug. 2015).

[13] Tsuji, T., "Cool luminous stars: the hybrid nature of their infrared spectra - Carbon, oxygen, and their isotopic abundances in $23 \mathrm{~K}$ - M giant stars," Astronomy and Astrophysics 489, 1271-1289 (Oct. 2008). arXiv: 0807.4387.

[14] Ohnaka, K., "Spatially resolving the inhomogeneous structure of the dynamical atmosphere of Betelgeuse with VLTI/AMBER.," Memorie della Societa Astronomica Italiana 80, 715 (2009).

[15] Smith, N., Hinkle, K. H., and Ryde, N., "Red Supergiants as Potential Type IIn Supernova Progenitors: Spatially Resolved 4.6 m CO Emission Around VY CMa and Betelgeuse," The Astronomical Journal 137, 3558-3573 (Mar. 2009).

[16] Lobel, A., Dupree, A. K., and Gilliland, R. L., "Spatially resolved tomographic STIS spectroscopy of Betelgeuse," 195, 45.03 (Dec. 1999).

[17] Lobel, A., Aufdenberg, J., Dupree, A. K., Kurucz, R. L., Stefanik, R. P., and Torres, G., "Spatially Resolved STIS Spectroscopy of Betelgeuse's Outer Atmosphere," 219, 641 (Jan. 2004).

[18] Norris, B. R. M., Tuthill, P. G., Ireland, M. J., Lacour, S., Zijlstra, A. A., Lykou, F., Evans, T. M., Stewart, P., and Bedding, T. R., "A close halo of large transparent grains around extreme red giant stars," Nature 484, 220-222 (Apr. 2012).

[19] Norris, B., Schworer, G., Tuthill, P., Jovanovic, N., Guyon, O., Stewart, P., and Martinache, F., "The VAMPIRES instrument: imaging the innermost regions of protoplanetary discs with polarimetric interferometry," Monthly Notices of the Royal Astronomical Society 447, 2894-2906 (Mar. 2015).

[20] Ireland, M. J., Scholz, M., and Wood, P. R., "Dynamical opacity-sampling models of Mira variables - II. Time-dependent atmospheric structure and observable properties of four M-type model series," Monthly Notices of the Royal Astronomical Society 418, 114-128 (Nov. 2011).

[21] Sharp, R. and Birchall, M. N., "Optimal Extraction of Fibre Optic Spectroscopy," Publications of the Astronomical Society of Australia 27, 91-103 (Mar. 2010). 